\title{
Nurses' Opinion About Euthanasia in East Azerbaijan Province, Iran
}

\author{
Rabee Rahimi ${ }^{1, *}$; Azad Rahmani ${ }^{2}$; Vahid Pakpour ${ }^{3}$; Narges Moradi ${ }^{2}$; Asghar \\ Mohammadpoorasl ${ }^{4}$; Zahra Pashaie ${ }^{5}$ \\ ${ }^{1}$ Nursing Department, Islamic Azad University, Bonab Branch, Bonab, IR Iran \\ 2 Department of Medical Surgical Nursing, Faculty of Nursing and Midwifery, Tabriz University of Medical Sciences, Tabriz, IR Iran \\ ${ }^{3}$ Student Research Committee, Community Health Nursing Department, Tabriz University of Medical Sciences, Tabriz, IR Iran \\ ${ }_{5}^{4}$ Department of Human Nutrition and Food Safety, School of Public Health, Qazvin University of Medical Sciences, Qazvin, IR Iran \\ ${ }_{5}^{5}$ Medical-Surgical Nursing Department, Maragheh Nursing and Midwifery Faculty, Maragheh, IR Iran \\ *Corresponding author: Rabee Rahimi, Nursing Department, Islamic Azad University, Bonab Branch, Bonab, IR Iran. Tel: +98-4127221935, Tel.: Email: laleh.rahimi3@gmail.com
}

Received: August 17, 2014; Revised: May 25, 2015; Accepted: June 5, 2015

\begin{abstract}
Background: Euthanasia is one of the important challenges in the field of end of life care. There are few studies investigated the attitude of Shiite Muslim nurses about different aspects of euthanasia.

Objectives: The aim of present study was to investigate the attitude of Iranian Shiite nurses about different aspects of euthanasia in East Azerbaijan Provence, Iran, 2012.

Patients and Methods: In this descriptive study, 209 nurses (census sampling method) from 6 educational hospitals in East Azerbaijan Province were participated. The attitude of nurses was investigated with Euthanasia Attitude Questionnaire, which is a 31-item scale. This scale investigate the attitude of nurses in 5 domains, including general attitude ( 3 items), legal and religious issues ( 5 items), end of life care (8 items), euthanasia decision making ( 8 items), and attitude toward different types of euthanasia ( 7 items). Descriptive statistics were used for data analysis using SPSS software (ver. 13).

Results: Participants had a negative attitude towards all aspects of euthanasia. They reported that theywould not participate in euthanasia procedure even this procedure was accepted by religious leaders and legal authorities. They believed that caring of end of life patients is a burden but this is not a reason for euthanasia and also they did not consider patients or their relative as decision makers for euthanasia. In addition, participants rejected all types of euthanasia.

Conclusions: Iranian nurses in East Azerbaijan Provence have negative attitudes toward different aspects of euthanasia and this negative attitude is not related to religious or legal issues. So, searching for the reasons for such an attitude needs more investigation.
\end{abstract}

Keywords: Palliative Care; Euthanasia; Iran; Nurses

\section{Background}

Advancement in medical care has resulted in considerable progress in prolonging human life and highlights the end of life issues such as euthanasia (1). Euthanasia comes from the Greek word euthanatos, which means good death (2). Euthanasia is the purposeful ending of incurable patients' life (3).

Euthanasia may be divided into active and passive types. Active euthanasia is a planned and purposeful intervention for causing immediate death in terminal patients with no hope for cure. On the other hand, passive euthanasia is the intentional withholding or withdrawing of medical treatments or equipment that aimed to prolong the life of incurable patients (4). Also, euthanasia may be voluntary, when the patient asks to be killed, and involuntary, when the incapable patient is killed without his or her consent, and non-voluntary, when a capable patient is killed without hisher consent (5).

One important group of healthcare personnel that their viewpoint about 'end of life' issue, including euthanasia, is important is nurses. However, the viewpoints of nurses about euthanasia are rarely heard (6). There are some studies investigated the attitude of nurses about euthanasia. The results of some studies in Western countries, such as Belgium (7-9), Australia (10), and Sweden (11) showed that most nurses in these countries agreed with legalization of euthanasia. On one hand, some studies in Asian countries, especially Japan $(12,13)$ and Malaysia (14) showed negative attitude of healthcare professionals, including nurses, about euthanasia. On the other hand, one study in India showed that $69 \%$ of Indian physicians approve the euthanasia (15).

Religion and cultural background are important factors in determining the attitude about euthanasia (15). Islam emphasizes the significant value of human life and highlights the accountability of each human being for his or her own body. In Islam, euthanasia is considered a

Copyright (C) 2015, Ahvaz Jundishapur University of Medical Sciences. This is an open-access article distributed under the terms of the Creative Commons Attribution-NonCommercial 4.0 International License (http://creativecommons.org/licenses/by-nc/4.0/) which permits copy and redistribute the material just in noncommercial usages, provided the original work is properly cited. 
violation of the sacredness (16), because Muslims believe that only God has the authority to call back the soul (17). As a result, euthanasia is considered the same as suicide and regarded as an unforgiveable sin and the person who wants euthanasia and those aiding him or her are considered as sinners (18).

There are few studies in Muslim countries aimed to investigate the viewpoint of Muslim nurses about euthanasia. In two studies, 33\% of Turkish nurses $(19,20)$ reported that euthanasia should be legal in some circumstances. It should be noted that most Turkish people are Sunni Muslims which is different from Shiite Muslims who are the majority of people in Iran. There are two studies investigated the attitude of Iranian nurses about euthanasia. These studies showed that about $50 \%$ of Iranian nurses approve euthanasia, especially its voluntary form $(21,22)$. However, in these studies only the viewpoint of nurses about different types of euthanasia were investigated and other issues related to euthanasia such as attitude of nurses regarding legal and religious issues, end of life care, and euthanasia decision making were not investigated. To our view, this information is vital for further clarification of healthcare providers' attitude regarding euthanasia.

\section{Objectives}

The present study aimed to investigate the attitude of Iranian Shiite nurses about different aspects of euthanasia in East Azerbaijan Provence, Iran, 2012.

\section{Patients and Methods}

This descriptive study was conducted between June and November 2012. Six educational hospitals in East Azerbaijan province (EAP), a northwestern province in Iran, were selected as a setting for the study. All of these hospitals are affiliated to Tabriz University of Medical Sciences. This university is the main institute for providing health care services in EAP.

The sample of the study consisted of all nurses who were working in these hospitals during the sampling period. Inclusion criteria were as follows: having BS or MS in nursing, having at least 6 months experience in providing nursing care, and willingness to participate in the study. The exclusion criteria for the study consisted of having management positions and being apart from Azeri cultural background and Shiite Muslim sect. The sample size of 197 nurses was estimated based on a pilot study. All of nurses who meet the inclusion criteria were 232 nurses, so all of them were invited to participate in the study.

A questionnaire with two parts was used for data collection. The first part was assessed some demographic and professional characteristics of nurses. Second part was Euthanasia Attitude Questionnaire (EAQ). EAQ is a 31-item questionnaire that investigated the viewpoints of participants towards euthanasia in 5 domains, including general attitude (3 items), legal and religious issues (5 items), end of life care ( 8 items), euthanasia decision making ( 8 items), and attitude toward different types of euthanasia (7 items). Each item is rated on a 5-point Likert scale, ranges from definitively agree (score 5), agree (score 4), undecided (score 3 ), disagree (score 2), to definitely disagree (score 1) (20).

After obtaining permission, EAQ was translated into Persian by a translator and back translate procedure was conducted by two independent English-Persian translators. Then, the translated questionnaire was content validated by 15 academic staff from Tabriz university of medical sciences (TUOMS). It should be noted that in this stage one item was added to the questionnaire. The reliability of translated questionnaire was determined using Cronbach $\alpha$ coefficient, after pilot study on 20 nurses ( 0.89 for total questionnaire).

The research project was approved by regional ethics committee at Bonab branch of Islamic Azad University (ethical code $=92-15$ ) and the list of all potential participants was obtained from the nursing office of each hospital. Then, two researchers contacted all selected nurses and after informing nurses about the aims and methodology, their informed consents were obtained. At that time, the study questionnaire was given to all participants and some guidance was given to them before completion of the questionnaire. One week later, the researchers met all participants to gather the completed questionnaires. Nurses who did not return the questionnaire in one week were invited again to participate in the study. Finally, during 4 month of data collection, 232 nurses were invited and the data of 209 of them were collected (response rate $=90 \%$ ).

The data analysis was performed using the statistical program SPSS (version 13, Chicago, IL, USA). Frequency, percentage, mean, and standard deviation were used to describe some characteristics of nurses and their viewpoint about euthanasia.

\section{Results}

Some demographic and related professional characteristics of participants are presented in Table 1. As evident in the Table 1 , most participants were female, married, educated at baccalaureate degree and employed in Intensive Care Units. Also, most of the participants were aware that euthanasia is illegal in Iran and against the Islam rules.

The responses of participants to all items of EAQ are reported in Table 2. Regarding legal and religious domain, nurses have negative attitude towards main items. For example, $77.5 \%$ of nurses reported that even if euthanasia became legalized they would not engage in it and $71.7 \%$ of them reported that even by acceptance of euthanasia by religious leaders they would not engage in euthanasia procedure (Table 2 ). 
Rahimi Ret al.

Table 1. Some Demographic and Related Professional Characteristics of Participants ${ }^{\text {a }}$

\begin{tabular}{|c|c|}
\hline Characteristics & Values \\
\hline \multicolumn{2}{|l|}{ Gender } \\
\hline Male & $31(15.4)$ \\
\hline Female & $170(84.6)$ \\
\hline \multicolumn{2}{|l|}{ Marital status } \\
\hline Single & $55(27)$ \\
\hline Married & $149(73)$ \\
\hline \multicolumn{2}{|l|}{ Degree } \\
\hline BS & $185(93.9)$ \\
\hline MS & $12(6.1)$ \\
\hline \multicolumn{2}{|l|}{ Ward } \\
\hline Medical & $64(33)$ \\
\hline Surgical & $46(23.7)$ \\
\hline Intensive care units & $84(43.3)$ \\
\hline \multicolumn{2}{|l|}{ Euthanasia is illegal in Iran } \\
\hline Yes & $192(91.9)$ \\
\hline No & $17(8.1)$ \\
\hline \multicolumn{2}{|c|}{ Euthanasia is against the Islam rules } \\
\hline Yes & $203(97)$ \\
\hline No & $6(3)$ \\
\hline Age, y & $32.6 \pm 7.1$ \\
\hline Experience in nursing, $y$ & $8.8 \pm 7.0$ \\
\hline
\end{tabular}

With regard to 'end of life care' domain, most nurses reported that care of a patient in terminal stage is a burden for relatives, health care system, and medical staff. Also, most nurses disagreed with ending the life of persistent vegetative patients, patients without hope, and patients with incurable diseases. In addition, most nurses disagreed with do-not-resuscitate order (DNR). It means that regardless of awareness about burden of end of life care, most of nurses were against the ending the life of terminal patients (Table 2).

Regarding 'decision making' domain, most nurses disagreed about engaging families in euthanasia decision for incapable or unconscious patients and believed that even patients with an incurable disease should not be allowed for euthanasia decision. In addition, they believed that euthanasia decision should not be applied for incapable patients. These key items show a negative attitude of nurses about euthanasia decision-making (Table 2).

With respect to 'attitude toward different types of euthanasia' domain, nurses reported their negative attitude and most of them disagreed with active and passive euthanasia (Table 2). Regarding general attitude domain, most nurses believed that application of euthanasia decreases the confidence of public to medicine and they would not like to apply euthanasia for themselves or their families.
Also, most of them believed that euthanasia is the killing of a person, not helping him/her to die (Table 2).

\section{Discussion}

According to extensive review of the related literature, this study is the first that investigated the viewpoint of Shiite nurses in Iran about different aspects of euthanasia. In general, these nurses have negative attitudes towards different aspects of euthanasia procedure.

As mentioned before, there are few studies investigated the viewpoint of Muslim nurses about euthanasia. In two previous studies, nearly one-third of Turkish nurses $(19,20)$ reported that euthanasia should be legal only in some circumstances. However, there are other studies investigated the attitudes of medical staff in Muslim countries. In one study, only $25 \%$ of Pakistani medical students approved the legalization of euthanasia (23). In another study, most of Pakistani medical students did not approve the euthanasia and believed that this procedure should be illegal in their country (24). Similarly, in other studies most of Sudanese doctors reported that they were against euthanasia and not willing to legalize this procedure in their country (25). All of these studies were in agreement with the findings of the present study and showed that Muslim health care providers have a negative attitude towards euthanasia.

In legal and religious domain, participants reported that they will not engage in euthanasia procedure even if it is approved by religious leaders or legal authorities. As mentioned before, according to all Islamic sects, all types of euthanasia are forbidden and this procedure considered an unforgiveable sin (18). Similarly, all types of euthanasia are illegal in Iran (21). But, the result of the present study is interesting and showed that religious or legal issues are not the main reason for negative attitudes of Iranian nurses about euthanasia. Also, most participants of the present study reported that they know that euthanasia is illegal in Iran and is against the Islamic rules. This finding is important but investigating the reasons for such attitude needs more studies.

With regard to 'end of life care' domain, most nurses believed that care of end of life patients is a burden for family, medical staff, and nurses. But, in spite of this awareness, most of them disagreed with ending the life of these patients and even were against DNR orders. Similarly, in another study, most Iranian nurses reported that they tended to care end of life patients and considered death as a natural part of life and were not likely to speak about death with patients (26). Another study showed that Iranian nurses believed that their main responsibility is to save patients' life and do not like to participate in any procedures that their aims are to end the life of patients (17). About DNR orders, two Iranian studies showed that most Iranian nurses (27) and Iranian nursing students (28) disagree with DNR orders. This finding confirms the results of the present study. 


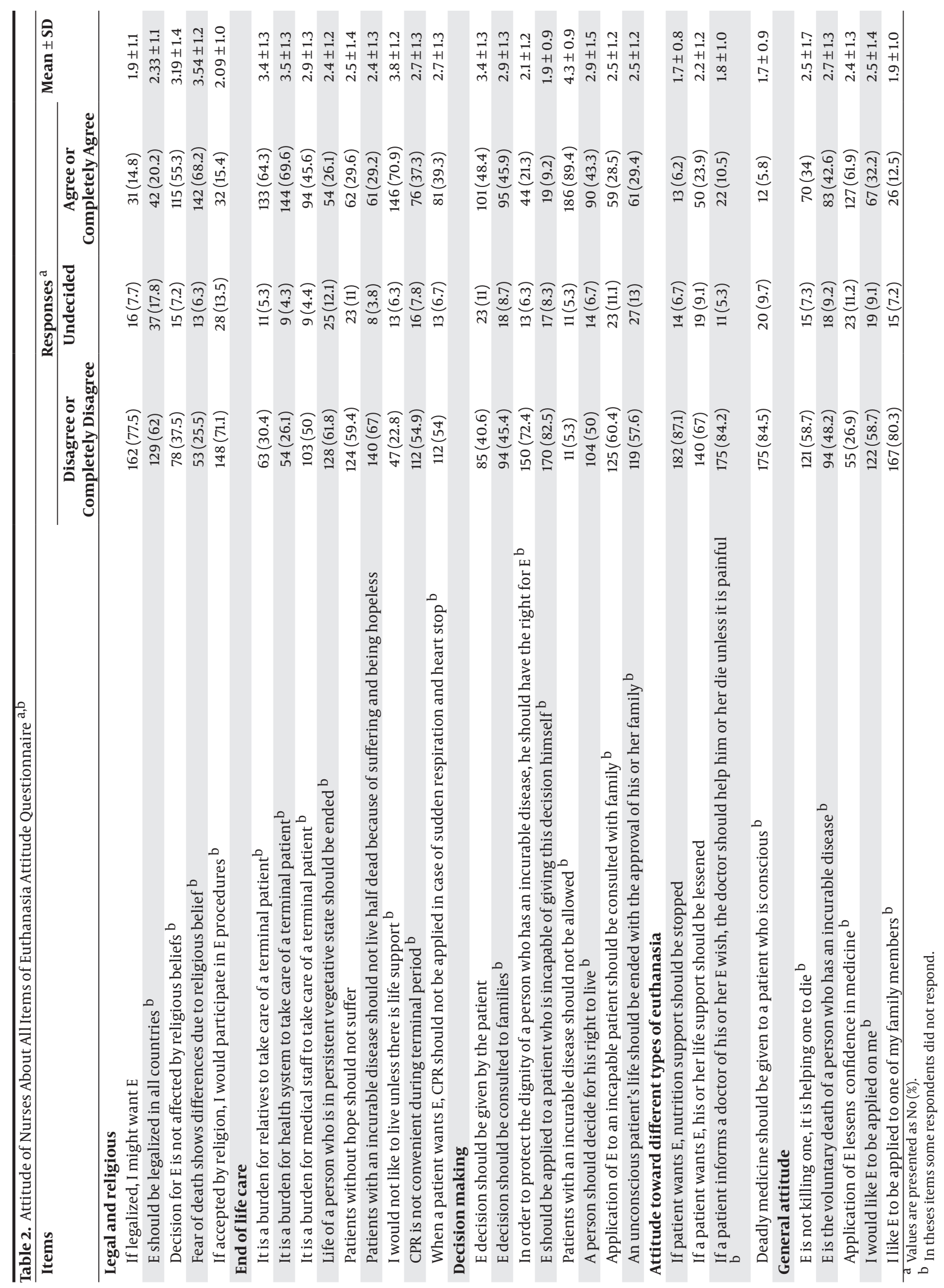


Participants showed their negative attitude about different types of euthanasia, including active and passive forms. Pervious Iranian studies showed that nearly $50 \%$ of Iranian nurses agreed with euthanasia, especially passive form of it $(21,22)$. It should be noted that these studies were conducted in different cultures in Iranian context and may indicate that cultural issues are very important in attitude of health care professionals towards different types of euthanasia. In this regard, previous multicultural study showed that, Iranian nursing students in comparison with Swedish nursing students, were more afraid of death and less inclined to deliver the care for dying persons (29). Also, approving the role of cultural background in attitudes toward different types of euthanasia needs more studies.

The results of the present study have some clinical implications. As it was shown, most participants disagreed with different aspects of euthanasia even with passive euthanasia or euthanasia in specific circumstances. So, this attitude is an important barrier for legalization of euthanasia or related procedures and should be considered in legalization process.

This study has some limitations too. First, the study population were the nurses of 6 educational hospitals on East Azerbaijan Province in northwest of Iran and do not cover the cultural and religious diversity of Iran. Secondly, nurses from private hospitals were not included in this study. Thirdly, because of the sensitivity of the study topic, some nurses might be reluctant to report their real attitude. So, more studies are required for further clarification of the attitude of Iranian nurses about euthanasia. Other studies in different cultures of Iran or other cultures in the Middle East countries are needed too. Moreover, investigating the reasons for negative attitude of Iranian nurses toward euthanasia needs more studies. Finally, there is a need for more studies on nurses in private hospitals.

\section{Acknowledgements}

We appreciate all nurses who were participated in this study.

\section{Authors' Contributions}

Rabee Rahimi: Data collection and proposal writing; Azad Rahmani: Study management, proposal writing, and article writing; Vahid Pakpour: Data collection, article writing, and proposal writing; Narges Moradi: Data collection and article writing; Asghar Mohammadpoorasl: Statistical analysis; and Zahra Pashaie: Data collection and article writing.

\section{Funding/Support}

This study was supported by Research Deputy of Islamic Azad University, Bonab branch (Ethical code = $92-15)$.

\section{References}

1. Berghs M, Dierckx de Casterle B, Gastmans C. The complexity of nurses' attitudes toward euthanasia: a review of the literature. $J$ Med Ethics. 2005;31(8):441-6.

2. Bosek MSD, Savage TA. The ethical component of nursing education: Integrating ethics into clinical experience.Philadelphia: Lippincott Williams and Wilkins;2007.

3. Junger S, Payne S, Brearley S, Ploenes V, Radbruch L. Consensus building in palliative care: a Europe-wide delphi study on common understandings and conceptual differences.J Pain Symptom Manage. 2012;44(2):192-205.

4. Butts JB, Rich K. Nursing ethics: Across the curriculum and into practice.USA: Jones and Bartlett; 2012.

5. Steck N, Egger M, Maessen M, Reisch T, Zwahlen M. Euthanasia and assisted suicide in selected European countries and US states: systematic literature review. Med Care. 2013;51(10):938-44.

6. Brzostek T, Dekkers W, Zalewski Z, Januszewska A, Gorkiewicz M. Perception of palliative care and euthanasia among recently graduated and experienced nurses. Nurs Ethics. 2008;15(6):761-76.

7. Verpoort C, Gastmans C, Dierckx de Casterle B. Palliative care nurses' views on euthanasia. J Adv Nurs. 2004;47(6):592-600.

8. Inghelbrecht E, Bilsen J, Mortier F, Deliens L. Nurses' attitudes towards end-of-life decisions in medical practice: a nationwide study in Flanders, Belgium. Palliat Med. 2009;23(7):649-58.

9. Gielen J, van den Branden S, van Iersel T, Broeckaert B. Flemish palliative care nurses' attitudes toward euthanasia: a quantitative study. Int J Palliat Nurs. 2009;15(10):488-97.

10. Kitchener BA. Euthanasia and professional conduct. AustNurs Midwifery J. 2014;21(8):54-9.

11. Gielen J, van den Branden S, Broeckaert B. Religion and nurses' attitudes to euthanasia and physician assisted suicide. Nurs Ethics. 2009;16(3):303-18.

12. Asai A, Ohnishi M, Nagata SK, Tanida N, Yamazaki Y. Doctors' and nurses' attitudes towards and experiences of voluntary euthanasia: survey of members of the Japanese Association of Palliative Medicine. J Med Ethics. 2001;27(5):324-30.

13. Ozcelik H, Tekir O, Samancioglu S, Fadiloglu C, Ozkara E. Nursing students' approaches toward euthanasia. Omega (Westport). 2014;69(1):93-103.

14. Adchalingam K, Kong WH, Zakiah MA, Zaini M, Wong YL, Lang CC. Attitudes of medical students towards euthanasia in a multicultural setting. Med J Malaysia. 2005;60(1):46-9.

15. Kamath S, Bhate P, Mathew G, Sashidharan S, Daniel AB. Attitudes Toward Euthanasia Among Doctors in a Tertiary Care Hospital in South India: A Cross Sectional study. Indian J Palliat Care. 2011;17(3):197-201.

16. McDougall JF, Gorman M. Euthanasia: A Reference Handbook.Philadelphia: Lippincott Williams \& Wilkins; 2008.

17. Boroujeni AZ, Mohammadi R, Oskouie SF, Sandberg J. Iranian nurses' preparation for loss: finding a balance in end-of-life care. JClin Nurs. 2009;18(16):2329-36.

18. Aghababaei N, Wasserman JA. Attitude toward euthanasia scale: psychometric properties and relations with religious orientation, personality, and life satisfaction. Am J Hosp Palliat Care. 2013;30(8):781-5.

19. Kumas G, Oztunc G, Nazan Alparslan Z. Intensive care unit nurses' opinions about euthanasia. Nurs Ethics. 2007;14(5):637-50.

20. Turla A, Ozkara E, Ozkanli C, Alkan N. Health professionals' attitude toward euthanasia: a cross-sectional study from Turkey. Omega (Westport). 2006;54(2):135-45.

21. RastgariNajafabadi H, Sedagat M, SaidiTehrani S, Aramesh K. [Study of Tehran University of Medical Sciences hospitals nurses' attitudes regarding euthanasia]. Iranian Journal of Medical Ethics and History of Medicine. 2010;5(3):37-44.

22. Moghadas T, Momeni M, Baghaee M, Ahmadi S. [Euthanasia and care for dying patients: attitudes of ICU nurses]. Iranian Journal of Med Ethics and History of Med. 2012;5(3):75-83.

23. Shaikh MA, Kamal A. Beliefs about euthanasia among university students: perspectives from Pakistan. East Mediterr Health J. 2011;17(10):794-7.

24. Khan A, Hussain M, Hasan SM. Euthanasia: perspective of Paki- 
stani Medical students and practitioners-A survey. Professional Med J. 2012;19(1):101-6.

25. Ahmed AM, Kheir MM, Abdel Rahman A, Ahmed NH, Abdalla ME. Attitudes towards euthanasia and assisted suicide among Sudanese doctors. East Mediterr Health J. 2001;7(3):551-5.

26. Iranmanesh S, Dargahi H, Abbaszadeh A. Attitudes of Iranian nurses toward caring for dying patients. Palliat Support Care. 2008;6(4):363-9.

27. Mogadasian S, Abdollahzadeh F, Rahmani A, Ferguson C, Pakan- zad F, Pakpour V, et al. The attitude of Iranian nurses about do not resuscitate orders. Indian J Palliat Care. 2014;20(1):21-5.

28. Mogadasian S, Abdollahzadeh F, Rahmani A, Pakanzad F, Heydarzadeh H. Do not resuscitation order: attitude of nursing students of Tabriz and Kordestan University of Medical Sciences. Iranian Journal of Medical Ethics and History of Medicine. 2013;6(5):45-56.

29. Iranmanesh S, Axelsson K, Haggstrom T, Savenstedt S. Caring for dying people: attitudes among Iranian and Swedish nursing students. Indian J Palliat Care. 2010;16(3):147-53. 